P38 RESULTS FROM FIRST YEAR OF THE NHS'S FIRST TARGETED 'CHEMSEX' CLINIC IN GUM/HIV

David Stuart*, Alan McOwan. Chelsea and Westminster Hospital NHS Foundation Trust, 56 Dean Street GUM/HIV, London, UK

10.1136/sextrans-2015-052126.82

Background/introduction With much speculation and anecdotal reports regarding the causal links between sexualised recreational drug use by MSM (commonly referred to as 'ChemSex') and $\mathrm{HIV} / \mathrm{HCV}$ rates, there has been much demand from commissioners and researchers and practitioners to identify the extent of the problem. In 2014, one London GUM/HIV clinic launched the NHS' first targeted ChemSex clinic. This presentation includes robust data collected from 874 unique presentations in the first year of this landmark clinic.

Aim(s)/objectives The objective was to satisfy the health sector's concerns about the extent of this much hyped syndemic, with qualitative and quantitative data as well as assess interventions and cohort engagement methods.

Methods Targeted clinics and outreach services were established with skilled addiction staff and resourcing peer volunteers, collecting culturally and contextually appropriate behavioural trends and data.

Results Data includes:

- Effectiveness of certain contextually-appropriate questions re ChemSex during GUM consultation.

- ARV non-adherence amongst high-risk ChemSex party-goers who favour condomless sex.

- Condom use (or otherwise) and number of partners broken down to include HIV+ve MSM not on treatment.

- HIV/HCV broken down to include sexual acquisition versus injecting drug use acquisition.

- HCV data broken down to include number of re-infections amongst HIV-ve non-injecting drug users.

Discussion/conclusion This presentation includes the data, offers examples of how this model might be adapted in other services, and incorporates some training for attendees in how to overcome fears or ignorance regarding drug use risk assessments and consultations; it also includes film footage of role play exercises for skill-building purposes.

\section{P39 PROGRESSIVE MULTIFOCAL LEUKOENCEPHALOPATHY IN A HIV-POSITIVE PREGNANT WOMAN - FIRST CASE REPORTED IN THE UK}

Jun Yi Soh*, Siris Allan. Coventry and Warwickshire Partnership Trust, Coventry, UK

10.1136/sextrans-2015-052126.83

Background/introduction Progressive multifocal leukoencephalopathy, caused by the John Cunningham (JC) polyomavirus, is the third commonest cause of encephalopathy in HIV-infected patient and almost exclusively occurs in immunosuppressed individuals.

Case report A 33-year-old female presented with a late HIV diagnosis during antenatal period and commenced on combivir, darunavir and ritonavir. Baseline viral load was 168,051 copies/ $\mathrm{ml}$ and CD4 count was 70/100 $\mathrm{ml}$. She then developed right sided weakness and facial droop. Raltegravir was added at
33 weeks gestation. Power on right side was 4/5 with hyperreflexia and a mild cranial nerve VII palsy. Neurological examination done a week prior to presentation found no abnormalities.

Results Routine bloods were unremarkable. CSF analysis showed white cell count of $4 \times 10^{6} / \mathrm{L}$ predominantly lymphocytes, protein $0.59 \mathrm{~g} / \mathrm{L}$, polyomavirus DNA and JC virus DNA positive. Cryptococcal antigen, india ink stain and toxoplasma serology were negative. Initial MRI head showed multiple abnormal areas with the largest abnormal area seen in the left frontal lobe affecting white matter with extension into grey matter. Progress weakness worsened over the next 2 weeks, power was $1 / 5$ with expressive dysphasia. She was started on 5 days $1 \mathrm{~g}$ methylprednisolone which showed no improvement. Due to foetal distress on CTG, emergency caesarean was done at 36 weeks. Viral load at delivery was $<40$ copies $/ \mathrm{ml}$. Darunavir and ritonavir were stopped due to raised ALT, which resolved.

Discussion/conclusion PML progresses rapidly and prognosis is poor. Currently there are no treatment guidelines for PML and studies using mefloquine and methylprednisolone show inconclusive results.

\section{P40 PENILE SQUAMOUS CELL CARCINOMA IN A PATIENT TREATED WITH ETANERCEPT FOR PSORIASIS}

Beruwalage Swaris, Alison Wright*, Richard Inman, Sarah Cockayne. Sheffield Teaching Hospital, Sheffield, UK

\subsection{6/sextrans-2015-052126.84}

Background Differential diagnosis of genital ulceration includes benign skin disease, infection and malignancy. An increase in sexually transmitted infections in older patients has been seen. Biologics inhibit immune system components that fuel inflammation. They are used to treat refractory chronic inflammatory conditions, including psoriasis. Concerns have been raised regarding an association between use of biologic treatments such as Etanercept and squamous cell carcinoma (SCC). We present a case of a patient taking Etanercept for psoriasis, who developed penile SCC.

Case A 57 years old man, listed by Urology for biopsy of a penile ulcer of 8 week duration, was referred to Genitourinary Medicine to exclude infective causes. He had a long history of severe psoriasis which had been treated with Etanercept for more than 7 years. He had had sex with one casual female partner 3 months prior to presentation. A deep, clean, indurated ulcer was seen on the corona. Regional nodes were impalpable. Tests were negative for syphilis, HIV and herpes simplex. The biopsy showed invasive SCC. He subsequently underwent a partial penectomy.

Discussion Penile cancer is uncommon in the United Kingdom. Embarrassment may cause delay in presentation. Penile SCC has been reported in patients on Etanercept. Clear evidence of association is lacking. Patients on biologics should be advised to carefully examine their skin including the genitals, reporting any skin changes promptly. Increasing awareness among patients and physicians about this possible association could prevent delay in diagnosis. The National Biologic Registers will reveal more definite evidence over time. 